\title{
Notch1 induces enhanced expression of $\Delta$-like-1 in the U251MG glioma cell line
}

\author{
CHUN-FA QIAN ${ }^{1,2^{*}}$, WEI YAN ${ }^{1 *}$, JUN-XIA ZHANG $^{3}$, LEI SHI $^{4}$, JIN QIAN $^{1}$, \\ $\mathrm{ZHEN} \mathrm{FU}^{1}$, CHUN-SHENG KANG ${ }^{5}, \mathrm{NING} \mathrm{LIU}^{1}$ and YONG-PING YOU ${ }^{1}$
}

\author{
${ }^{1}$ Department of Neurosurgery, The First Affiliated Hospital of Nanjing Medical University, Nanjing 210029; \\ ${ }^{2}$ The Affiliated Brain Hospital of Nanjing Medical University, Nanjing 210029; ${ }^{3}$ Department of Neurosurgery, \\ Chinese Traditional Medical Hospital of Kunshan, Kunshan 215300; ${ }^{4}$ Department of Neurosurgery, Affiliated Kunshan \\ People's Hospital of Jiangsu University, Kunshan 215300; ${ }^{5}$ Department of Neurosurgery, Tianjin Medical University \\ General Hospital, and Laboratory of Neuro-Oncology, Tianjin Neurological Institute, Tianjin 300052, P.R. China
}

Received May 15, 2009; Accepted July 13, 2009

DOI: 10.3892/ijmm_00000251

\begin{abstract}
The Notch signaling pathway takes part in coordinated regulation of cell growth, survival and differentiation. Previous findings have shown that Notch1 and $\Delta$-like-1 (DLL1) are overexpressed in many glioma cell lines and primary human gliomas. Down-regulation of DLL1 by RNA interference inhibits proliferation and induces apoptosis in multiple glioma cell lines. Our studies showed that Notch1 expression plasmid induced more expression of DLL1 in the U251MG glioma cell line. Adversely, blocking Notch1 receptors down-regulated the expression of DLL1. Both down-regulating DLL1 and blocking Notch1 receptors induced U251MG cell apoptosis and proliferation inhibition, and combining the two treatments produced stronger effects than the sum of a single treatment. These findings suggest a positive feedback loop between Notch1 and DLL1, which may become an effective combined therapeutic target.
\end{abstract}

\section{Introduction}

Glioma is one of the most common primary brain tumors that has a grave prognosis. Standard therapies, such as surgery, chemotherapy and radiation, have been only modestly successful in treating patients with high-grade gliomas. It is hoped to explore more effective therapy targets through greater

Correspondence to: Dr Ning Liu, Department of Neurosurgery, The First Affiliated Hospital of Nanjing Medical University, Nanjing 210029, P.R. China

E-mail: nwliuning@126.com

Dr Yong-Ping You, Department of Neurosurgery, The First Affiliated Hospital of Nanjing Medical University, Nanjing 210029, P.R. China E-mail: yyp19@njmu.edu.cn

${ }^{*}$ Contributed equally

Key words: Notch signaling, glioma, Notch1, $\Delta$-like1, feedback loop knowledge of molecular signaling pathways involved in glioma cell proliferation and apoptosis.

The Notch signaling pathway determines cell fate by regulating cell differentiation, survival and apoptosis (1-3). For example, Notch signaling takes part in corneal epithelial differentiation and inversely correlates with a proliferative status (4). The role of Notch signaling in regulating differentiation and cell apoptosis indicates its potential effect on cancer cells. Dysregulation of Notch signaling leads to dramatic effects for the genesis and development of cancer (5). In human primary glioblastoma (GBM) samples and human GBM cell lines, Notch signaling is dysregulated. Additionally, inhibition of Notch signaling leads to differentiation and growth arrest of GBM cells (6).

In the Notch family, four Notch receptors and five ligands have been identified in mammals, including Notch1-4, Jagged1 and 2, $\Delta$-like (DLL) -1, -3 and -4 respectively, which are all single transmembrane proteins (7). Activation of the Notch signaling pathway needs direct cell-cell contact (8). When the Notch ligand expressed on an adjacent cell binds to the extracellular domain (NECD) of the Notch receptor, the receptor is exposed to proteolytic activation. Notch receptors cleave into the Notch intracellular domain (NICD) and NECD when the Notch ligand binds to membrane receptors. The NICD dissociates from NECD, translocates into the nucleus and binds to members of the CSL transcription factor family (CBF-1/Suppressor-of-hairless/Lag-1) which becomes a transcriptional activator and activates the large family of $\beta$ helix loop helix (bHLH) transcription factors, such as Hes and Hey genes (9), which regulate differentiation and cell survival. So the expression of the Notch ligand as the first beginning of the Notch signaling pathway is crucial for its activation.

Purow et al (10) found that Notch1 and its ligand, DLL1, were overexpressed in many glioma cell lines and primary human gliomas. Additionally, down-regulating DLL1 increased the apoptosis ratio of multiple glioma cell lines and inhibited proliferation. These findings showed, for the first time, the dependence of cancer cells on a single Notch ligand, DLL1, and suggested a potential juxtacrine loop between DLL1 and Notch1 in gliomas. On the other hand, previous studies found 
that Notch1 inhibited the $\Delta$ ligand expressed in neighbors in the development of the nervous system of Drosophila and vertebrates (11). To date, there is no data to show whether the expression of DLL1 is effected by Notch1 in glioma in the potential loop between Notch1 and DLL1. In this regard, we examined the role of Notch1 on DLL1 and found that Notch1 induces more expression of DLL1. Our findings implicate a positive feedback loop between Notch1 and DLL1 in the $\mathrm{U} 251 \mathrm{MG}$ cell line and the feedback loop is more effective joint therapeutic target.

\section{Materials and methods}

Cell culture. Human glioma cell line, U251MG, were purchased from the Chinese Academy of Sciences Cell Bank. U251MG cells were cultured in DMEM (Invitrogen) supplemented with $10 \%$ bovine serum albumin and maintained at $37^{\circ} \mathrm{C}$ in an atmosphere of $5 \% \mathrm{CO}_{2}$ and routinely passaged at 2- to 3-day intervals.

Notch1 plasmid transfection. Notch1 plasmid, which contains the active signaling portion of the Notch1 molecule (54767836 bp), was gifted by Xiasi Bio (Nanjing, China). We plated U251MG cells in six-well plates at a density of $4.5 \times 10^{4}$ per well and divided them into three groups, including a blank group, negative control group and Notch1 expression plasmid group with three replicate wells. After 24 h, Notch1 expression plasmid and control plasmid were transfected respectively. We verified the effect of Notch1 plasmid by Western blot analysis $48 \mathrm{~h}$ after transfection.

Notch1 receptors blocking. U251MG cells were plated in twelve-well plates at a density of $2.5 \times 10^{4}$. Cells were divided into five groups from $\mathrm{A}$ to $\mathrm{E}$ with three wells in each group. Anti-Notch1 goat polyclonal IgG (Santa Cruz Biotechnology, USA) was added at different dilutions 0, 1:20, 1:100, 1:250 and 1:500. After $2 \mathrm{~h}$ cultured in incubator, culture media was replaced and new media was added with donkey anti-goat IgG (Santa Cruz Biotechnology, USA) in corresponding double dilutions at $0,1: 250,1: 125,1: 50$ and 1:10. After $72 \mathrm{~h}$ total protein and RNA were extracted after trypsinization (12).

Immunofluorescence. Human glioma U251MG cells were cultured on coverslips at density $4.5 \times 10^{4}$ per well. After antiNotch1 goat polyclonal IgG (1:100, Santa Cruz Biotechnology, USA) was added and cultured in incubator for $2 \mathrm{~h}$ as described above, cells were fixed with $75 \%$ alcohol for $30 \mathrm{~min}$ at $4{ }^{\circ} \mathrm{C}$. This was followed by three washes in PBS and $30 \mathrm{~min}$ incubation in FITC-labeled secondary antibodies (1:50, Santa Cruz Biotechnology, USA). After three more washes with PBS, images were captured digitally in five random microscope fields from each sample with the appropriate software (AxioVision Rel. 4.5) under a fluorescence microscope (Zeiss Imager AI).

Western blot analysis. Cell lysate samples (30 $\mu \mathrm{g})$ from cultured cells were prepared using, subjected to 10 or $12 \%$ sodium dodecyl sulfate-polyacrylamide gel electrophoresis, and transferred to a polyvinylidene difluoride membrane (Millipore Corporation, USA). The membrane was blocked in 5\% nonfat milk and incubated with diluted antibodies against C-terminal Notch1 (1:100, Santa Cruz Biotechnology, USA) and DLL1 antibody (1:200, Santa Cruz Biotechnology, USA) overnight at $4^{\circ} \mathrm{C}$. Bound antibody was detected with horseradish peroxidase-conjugated secondary antibodies to goat $\operatorname{IgG}$ and rabbit $\operatorname{IgG}(1: 2,000$ and 1:1,500, respectively, Santa Cruz Biotechnology, USA) or GAPDH $(1: 2,000$, Kangchen Biotechnology, China) using ultra enhanced chemoluminescence Western blotting detection reagents (Multi Science Biotech, China). All antibodies were diluted in blocking buffer (3\% w/v nonfat dry milk, $20 \mathrm{~mol} / \mathrm{l}$ Tris-base, $100 \mathrm{mmol} / \mathrm{l} \mathrm{NaCl}$ ). Other buffers used during Western blotting were made following the Shinegene protocol. All Western bands were quantified by densitometry and are presented in the form of a bar graph.

Real-time PCR. RNA was extracted from human U251MG cells using Trizol reagent (Invitrogen, Carlsbad, USA). DLL1 expression levels in U251MG cells were measured by TaqMan-based real-time (RT)-PCR using SYBR PrimeScript RT-PCR Kit (Takara Biotec) on the ABI Prism 7300 HT Sequence Detection system (Applied Biosystem, Foster City, $\mathrm{CA}$ ), according to the manufacturer's instructions. The relative gene expression was calculated via a $2^{-\Delta \Delta \mathrm{Ct}}$ method (13). All samples were measured in triplicate. Primers were as follows (14), DLL1, forward primer 5'-TGTGTGACGAACACTACT ACGGAG-3', reverse primer 5'-GTGAAGTGGCCGAAG GCA-3'; ß-actin forward primer 5'-CCACACTGTGCCCA TCTACG-3', reverse primer 5'-AGGATATTCATGAGGTA GTCAGTCAG-3'.

DLL1 siRNA transfection. DLL1 small interfering RNA (siRNA) duplexes were constructed by GenePharma Company (Shanghai, China). Sequences were as follows (10), DLL1, forward primer 5'-GCCGACAAGAAUGGCUUCAUU-3', reverse primer 5'-UGAAGCCAUUCUUGUCGGCGC-3'; control, forward primer 5'-(UUCUCCGAACGUGUCA CGU)d(TT)-3', reverse primer 5'-(ACGUGACACGUUCGG AGAA) d (TT)-3'. U251MG cells were plated in six-well plates at a density of $4.5 \times 10^{4}$ per well and divided into three groups with three wells, respectively. Cells were transfected using Lipofectamine 2000 reagent (Invitrogen, Carlsbad, USA) at 70-80\% confluency. Transfection complexes were prepared according to the manufacturer's instructions and added directly to glioma cells to a final siRNA concentration of $10 \mathrm{nmol} / \mathrm{l}$. Transfection medium was replaced $8 \mathrm{~h}$ posttransfection.

Apoptosis assays. U251MG cells were seeded onto twelvewell plates at a density of $2.5 \times 10^{4}$ per well, transfected with DLL1 siRNA or preincubated with Notch1 antibody in 1:100 dilution, or two joint measures. Blank and negative control groups were set respectively. The apoptosis ratio was analyzed 72 h post-treatment using ApoScreen ${ }^{\text {TM }}$ AnnexinV-RPE Kit (Southern Biotech, Birmingham, USA) in accordance with the manufacturer's instructions. Annexin V-RPE and 7-Aminoactinomycin $\mathrm{D}$ (7-AAD) double stain was used to evaluate the percentages of apoptosis. Annexin $\mathrm{V}^{-}$and 7-AAD- cells were used as controls. Annexin $\mathrm{V}^{+}$and 7-AAD- cells were designated as apoptotic and Annexin $\mathrm{V}^{+}$and $7-\mathrm{AAD}^{+}$cells as necrotic (15). 
A

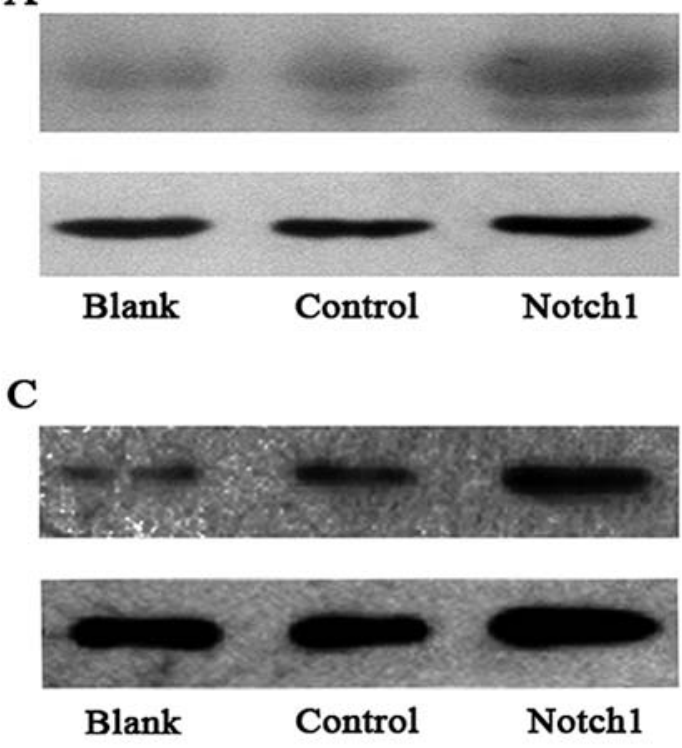

B

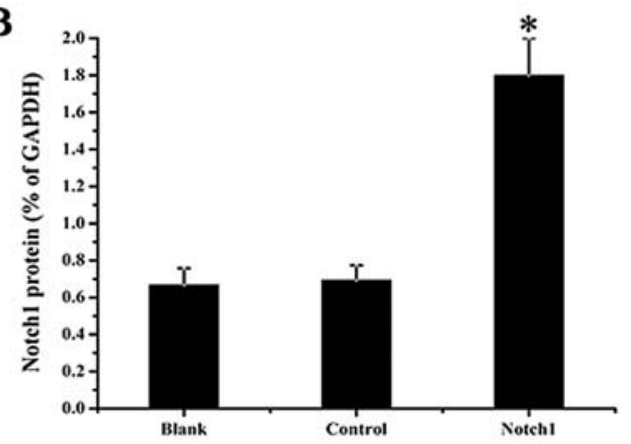

DLL1

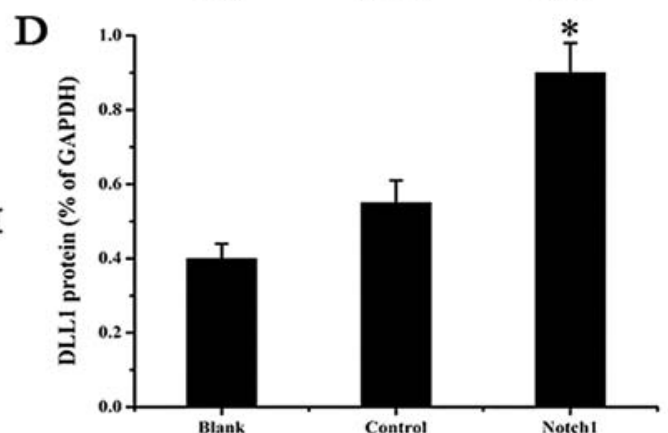

Figure 1. Western blot analysis of Notch1 and DLL1 expression. U251MG cells were transfected with Notch1 expression plasmid, negative control plasmid and no plasmid. In $48 \mathrm{~h}$, extracted total proteins were subjected to Western blot analysis with anti-Notch1 C-terminal portion IgG (A) and anti-DLL1 IgG (C) respectively. $(B, D)$ Columns, mean percents of special protein over GAPDH for triplicate wells; bars, \pm SE. Statistically significant differences from negative control were observed. ${ }^{*} \mathrm{P}<0.05$.
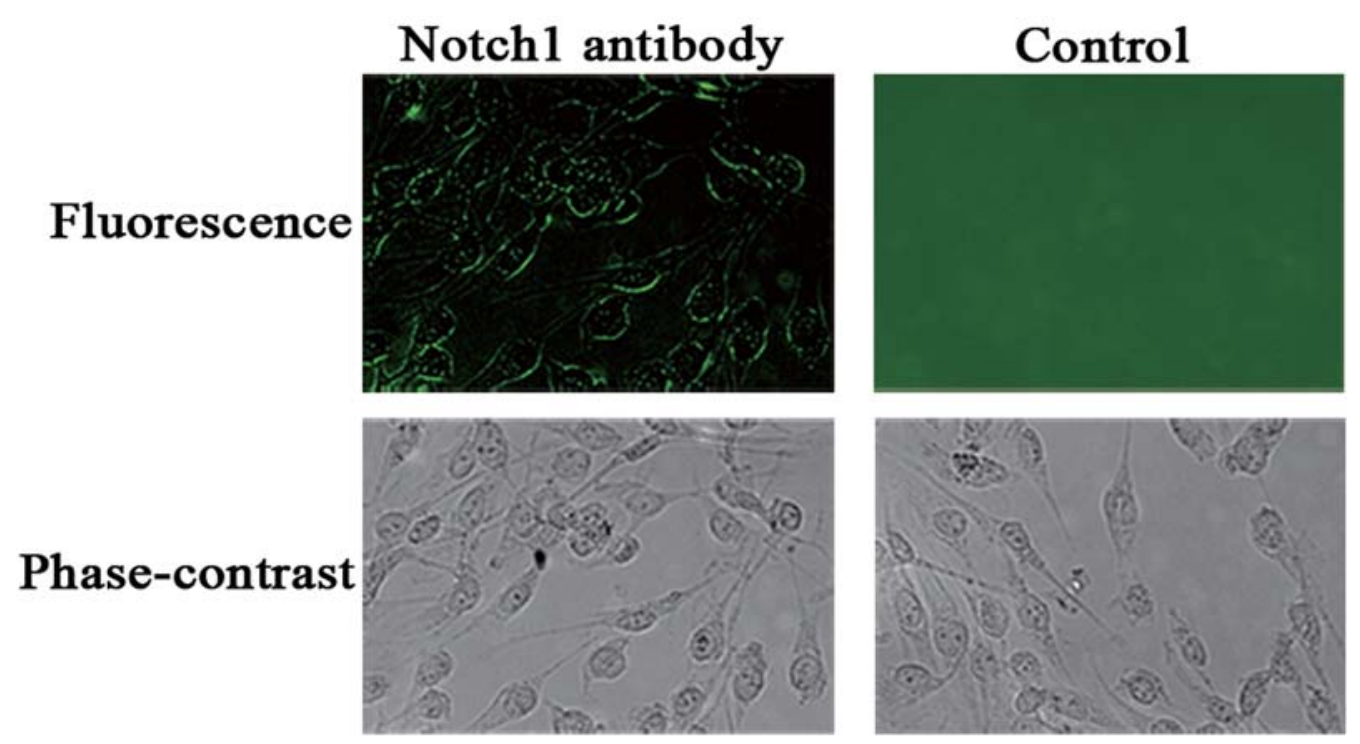

Figure 2. The binding effect of Notch1 antibody. U251MG cells were plated in twelve-well plates at a density of $2.5 \times 10^{4}$. Anti-Notch1 goat polyclonal IgG was added into culture media with 1:100 dilution, with PBS instead Notch1 antibody in control group. After 2 h cultured in incubator, the cells were fixed with $75 \%$ alcohol for $30 \mathrm{~min}$ at $4^{\circ} \mathrm{C}$. This was followed by three washes in PBS and 30 min incubation in FITC-labeled secondary antibodies (1:50). After three more washes with PBS, images were captured digitally in five random microscope fields from each sample with the appropriate software under a fluorescence microscope using blue light and white light as excitation light respectively. Under blue light, green fluorescence was seen on cell membrane in Notch1 antibody group while nonspecific fluorescence in control group, which illustrate Notch1 receptors were bound in Notch1 group indirectly. (Magnification, x100).

Cell growth assay. The WST-1 cell proliferation assays were used to determine relative cell growth as follows. U251MG cells were plated at $10^{4}$ cells per well in 96-well plates with six replicate wells for each condition, transfected with DLL1 siRNA or preincubated with Notch1 antibody, or two joint measures. Cell proliferation assays were performed $72 \mathrm{~h}$ later using WST-1 as described previously (16). The cell viability was determined at $450 \mathrm{~nm}$ absorbance using an enzymelinked immunosorbent assay plate reader. All data points represent the mean of six wells. 
Statistical analysis. All data were analyzed using SPSS Graduate Pack 11.0 statistical software (SPSS, Chicago, IL). Descriptive statistics including mean and \pm SE along with independent-sample t-test were used to determine significant differences. $\mathrm{P}<0.05$ was considered significant.

\section{Results}

Effects of transfection with Notch1 plasmid on DLL1. Our aim was to investigate whether Notch1 has the same effect on the expression of DLL1 in glioma as it does in the formation of the nervous system of Drosophila and vertebrates (11). Notch1 expression plasmid was transfected to U251MG cells and the expression of Notch1 and DLL1 was tested via Western blot analysis. As shown in Fig. 1A and B, $48 \mathrm{~h}$ after transfecting Notch1 expression plasmid, Notch1 was upregulated more than two times. Corresponding to Notch1, the expression of DLL1 was up-regulated in the Notch1 expression plasmid group nearly two times compared with negative control groups (Fig. 1C and D). So Notch1 plasmid induced enhanced expression of DLL1 while up-regulating Notch1.

Effects of blocking Notch1 receptors on DLL1. Notch1 expression plasmid induced more expression of DLL1 as described above. To further verify the effect of Notch1 on DLL1, we blocked Notch1 receptors by using anti-Notch1 goat polyclonal IgG and donkey anti-goat IgG consecutively (12). As shown in Fig. 2, in the Notch1 antibody group, epifluorescence is seen on the surface of most U251 cells while only nonspecific fluorescence appears in control group. This result shows that most Notch1 receptors are bound when the dilution of Notch1 antibody in culture media is up to $1: 100$. We performed RT-PCR and Western blot analysis after obstructing the site of Notch1 receptors with the antibody at different dilution levels. The expression of DLL1 was strongly down-regulated compared with the blank group. In addition, the expression level of DLL1 negatively correlated with the downward trend of the dilution of Notch1 antibody from low to high until 1:20 (Fig. 3). Notch1 antibody with 1:100 and 1:20 dilutions have similar effects, which demonstrates that the sites of Notch1 receptor are saturated when the dilution is up to $1: 100$. So we set $1: 100$ as the default dilution of Notch1 antibody in following experiments.

Transfection efficiency of DLL1 siRNA and effect on DLL1. Comparing random field of fluorescent microscope and corresponding light-phase microscope, $>80 \%$ U251 cells were seen by green fluorescent protein (GFP) reporter protein fluorescence $72 \mathrm{~h}$ after transfection (Fig. 4A and B), illustrating the transfection ratio of $>80 \%$. As shown in Fig. $5 \mathrm{~A}$ and $\mathrm{B}$, the level of DLL1 protein, normalized by GAPDH at the same membrane, was much lower in DLL1 siRNA group compared with blank and control groups, $\mathrm{p}<0.05$. Based on these results, DLL1 in U251 cells is knocked down successfully by transfecting DLL1 siRNA.

Effects of down-regulating DLL1, blocking Notch1 receptors and combined treatments on apoptosis and proliferation in U251MG glioma cells. Notch signaling pathway plays an

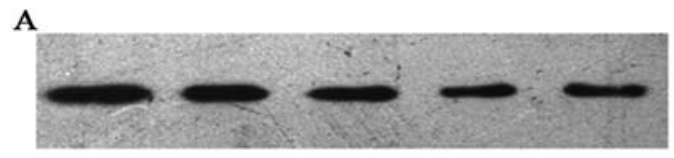

DLL1
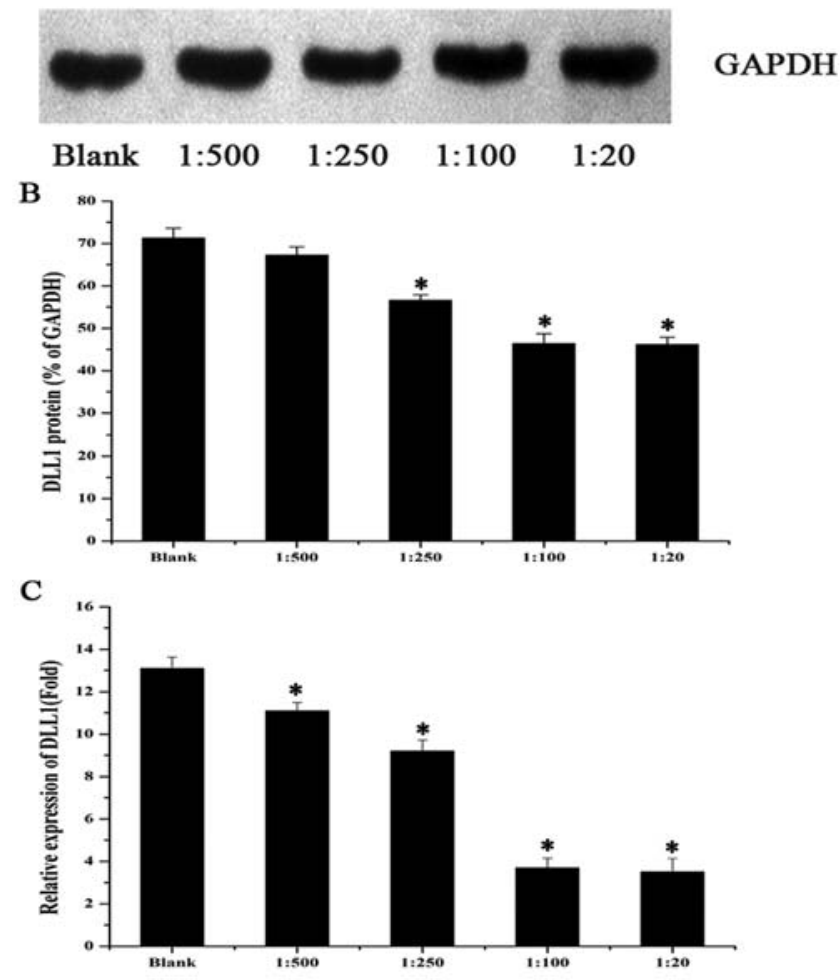

Figure 3. The effect of blocking Notch1 receptors on DLL1 expression level. U251MG cells preincubated with Notch1 antibody at different dilutions (0, 1:500, 1:250, 1:100 and 1:20). After $2 \mathrm{~h}$ culture media was replaced and new media was added with donkey anti-goat $\mathrm{IgG}$ in corresponding double dilutions at $0,1: 250,1: 125,1: 50$ and 1:10. U251MG cells were harvested for $72 \mathrm{~h}$. Western blot analysis (A) and RT-PCR (C) were done using total cell lysates and RNA respectively. Columns, mean percents of special protein over GAPDH for triplicate wells (B) and relative expression of DLL1 over $B$-actin $(C)$ bars, \pm SE. Statistically significant differences from negative control were observed; ${ }^{*} \mathrm{P}<0.05$.

integral role in determining cell fates, such as proliferation, differentiation and apoptosis. To investigate the effect of the juxtacrine loop between Notch1 and DLL1 on apoptosis in U251MG cells, we performed an apoptosis assay on U251MG cells using Annexin V-RPE and 7-AAD double staining after knocking down DLL1 by DLL1 siRNA, blocking Notch1 receptors and combined the two measures. The apoptosis ratio was compared with the blank group, the apoptosis induced by blocking Notch1 receptors was $13.52 \pm 1.27 \%$; compared with control siRNA group, knocking down DLL1 and the joint measure increased the proportion of apoptosis $15.15 \pm 1.38 \%$ and $41.32 \pm 1.97 \%$, respectively (Fig. 6A). The joint measure had a higher apoptosis ratio than either the treatment or the sum of the single treatment. These data show an important role of DLL1-Notch1 juxtacrine loop in apoptosis of U251MG cells. There is no statistical difference of apoptosis levels between knocking down DLL1 and blocking Notch1 receptors, which demonstrates that DLL1 and Notch1 were equally important in the juxtacrine loop. But combined blocking of Notch1 receptors and DLL1 siRNA caused much more apoptosis, which shows a 

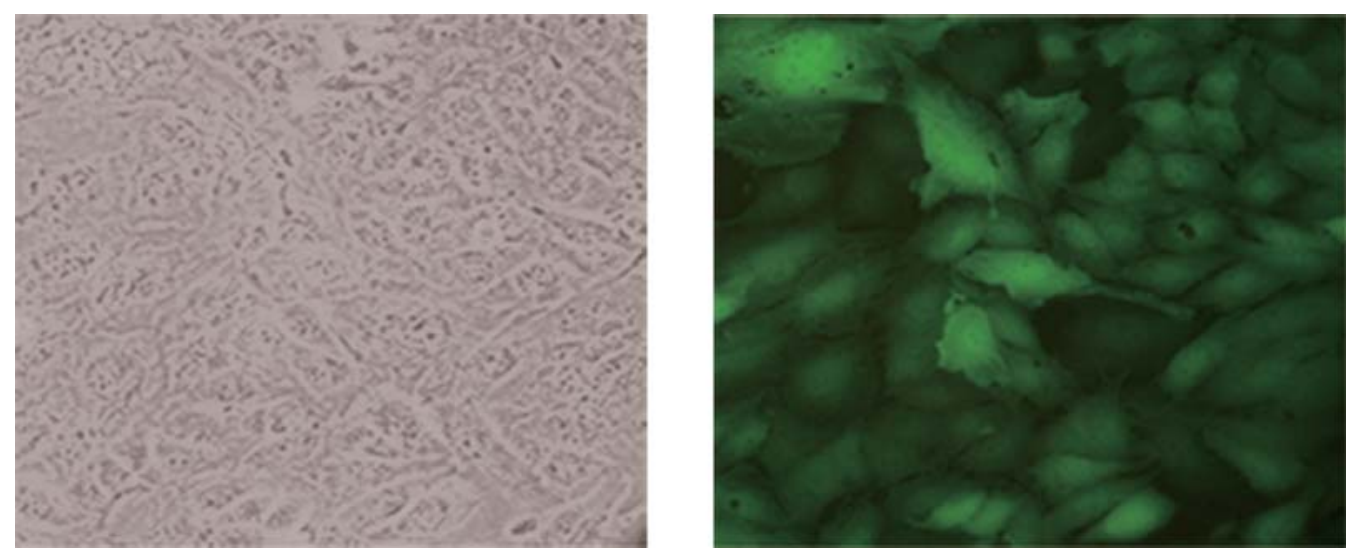

Figure 4. Image of green fluorescent protein (GFP) reporter protein fluorescence in U251MG cells to assess the percentage of positively transfected cells at $72 \mathrm{~h}$ post transfection. Phase-contrast images of cells are shown in the corresponding right-hand column. (Magnification, x100).

A

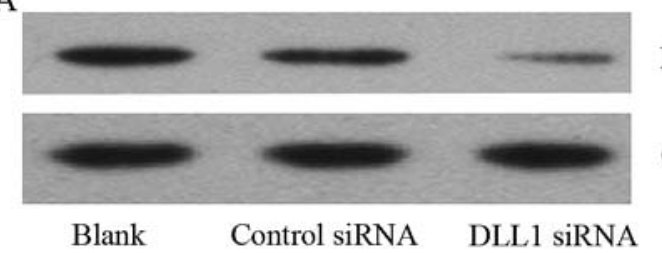

B

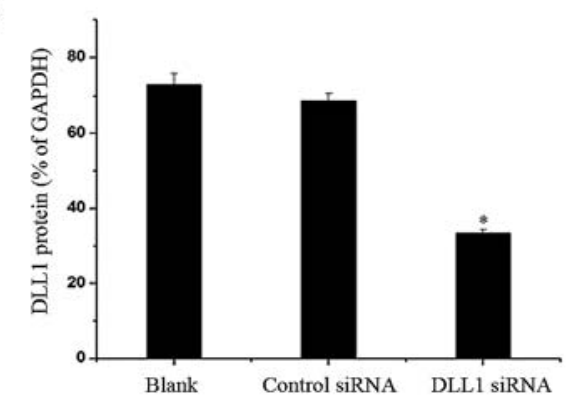

Figure 5. Western blot analysis of DLL1 after transfecting DLL1 siRNA. (A) U251MG cells cultured in twelve-well plates were divided into three groups. Besides one group as blank control, other two groups were pretreated with DLL1 siRNA and control siRNA. After $72 \mathrm{~h}$ total proteins extracted were subjected to Western blot analysis with anti-DLL1 IgG. (B) Columns, mean percents of DLL1 protein over GAPDH for triplicate wells; bars, \pm SE Statistically significant differences from negative control were observed; ${ }^{*} \mathrm{P}<0.05$.

potential synergistic reaction of blocking Notch1 receptors and down-regulating DLL1.

To investigate the importance of DLL1-Notch1 juxtacrine loop in U251MG glioma cell proliferation, we performed WST-1 cell proliferation and cytotoxicity assays after blocking Notch1 receptors, transfecting DLL1 siRNA and combining the two measures. Similarly to their effects on apoptosis, blocking Notch1 receptors and knocking down DLL1 resulted in inhibition of U251MG cell proliferation. The joint two measures produced a much stronger inhibition (Fig. 6B).

\section{Discussion}

Previous studies have shown that many glioma cell lines and primary human gliomas overexpress Notch1 and its ligand,
DLL1, which were demonstrated to be correlative on apoptosis and proliferation in multiple glioma cell lines (1). These findings suggest that glioma depends on a single Notch ligand and the existence of a potential Notch juxtacrine loop in gliomas. Additionally, previous studies have found that in the juxtacrine loop between the Notch receptor and $\Delta$ ligand, expression of Notch receptor inhibited $\Delta$ ligand of neighbors in the formation of the nervous system of Drosophila and vertebrates (11). To investigate whether Notch1 has the same effect on the expression of DLL1 in glioma, Notch1 plasmid was used to improve the expression level of Notch1. The effect of transfection was tested by Western blot analysis. The obvious up-regulation of Notch1 verified the result of Notch1 plasmid transfection. DLL1 was analyzed by Western blot analysis and RT-PCR after transfecting Notch1 plasmid. Here we firstly showed that the expression of DLL1 was upregulated after transfecting Notch1 plasmid in the U251MG glioma cell line, which shows a potential role of Notch1 in inducing more expression of DLL1.

To further verify the effect of Notch1 on DLL1, the sites of Notch1 receptors were obstructed using Notch1 and secondary antibodies with different dilutions. Our immunofluorescence result showed that most sites of Notch1 receptors were bound. The results of RT-PCR and Western blot analysis showed that DLL1 was strongly down-regulated when Notch1 receptors were blocked and correlated with the dilution of added antibody until 1:100. Blocking Notch1 receptors by Notch1 antibody prevented the activation of Notch1 receptors, and then weakened the role of Notch1 on DLL1 which indirectly downregulated the expression of DLL1. Notch1 enhanced the expression of DLL1, while DLL1 could bind to Notch1 receptors and induced Notch1 cleavage. In conclusion, there may be a positive feedback juxtacrine loop between Notch1 and DLL1 which plays an important role in the genesis and progression of glioma.

To testify the role of feedback loop between Notch1 and DLL1 in glioma, we down-regulated DLL1, blocked Notch1 receptors and combined the two measures, respectively. Further research showed that both down-regulation of DLL1 and blocking Notch1 induced apoptosis and growth inhibition in U251MG cells, which suggested that the juxtacrine loop between Notch1 and DLL1 played an important role in 

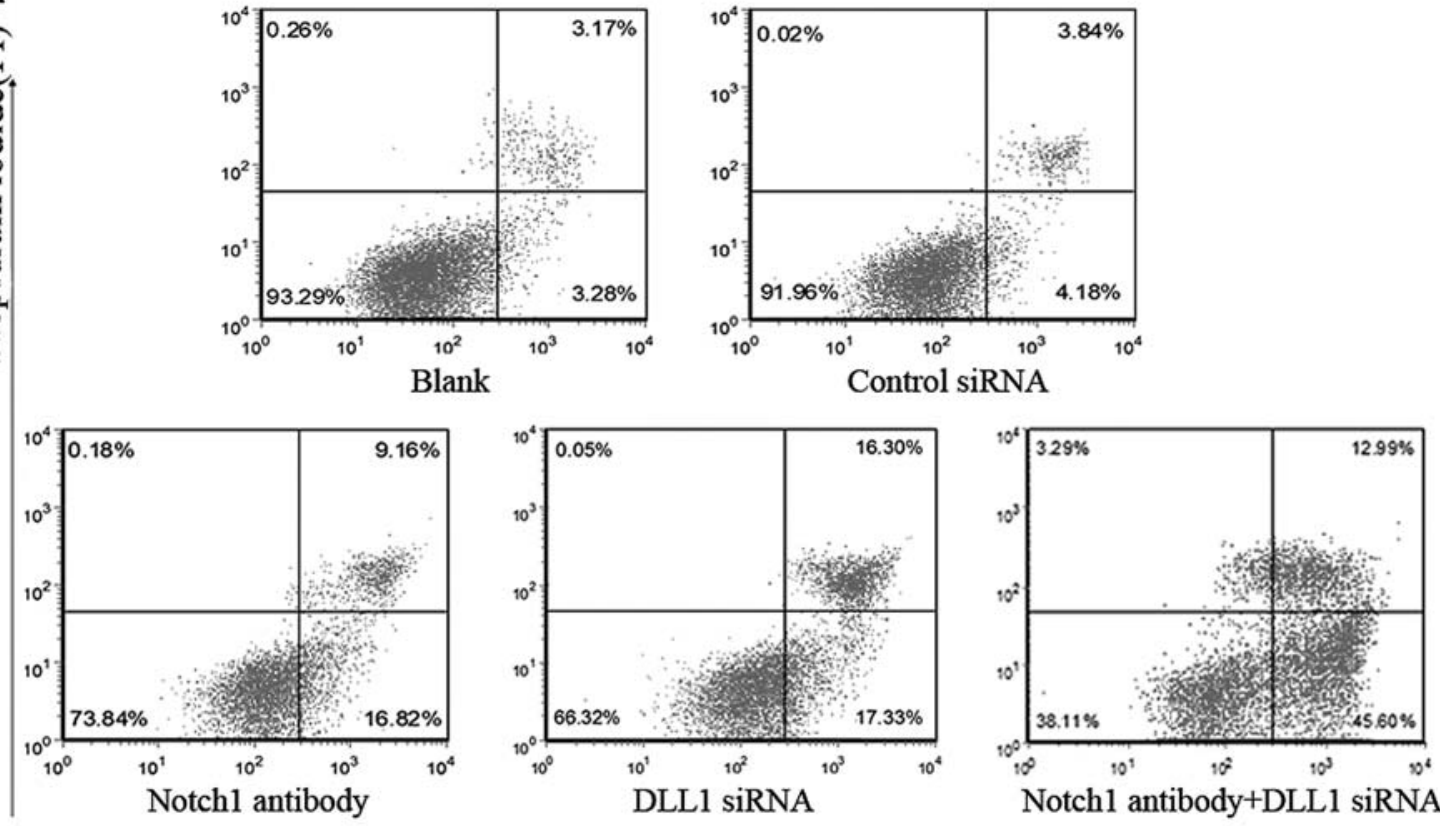

Notchl antibody+DLL1 siRNA

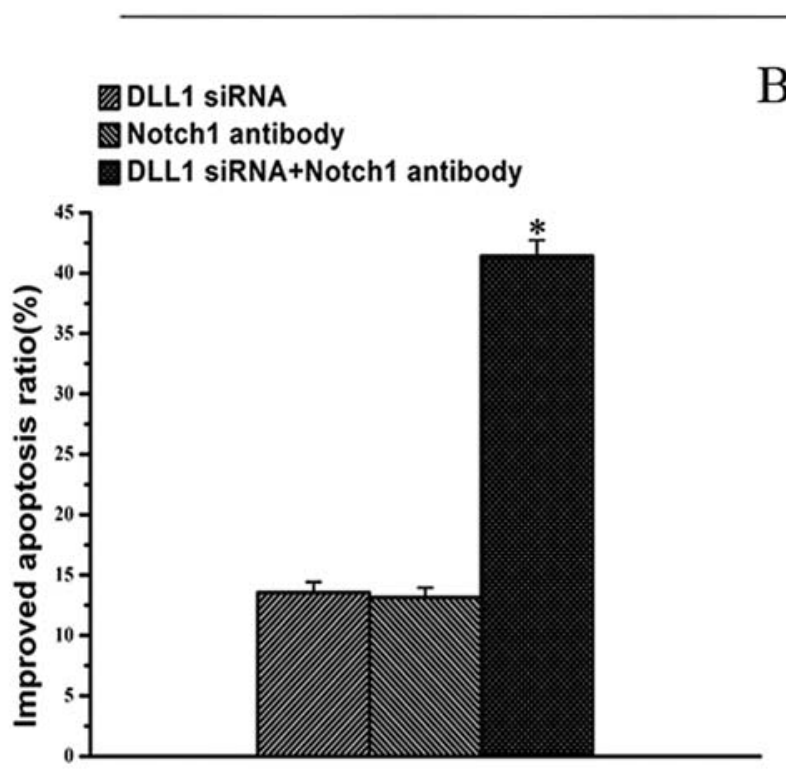

B

Annexin V-RPE
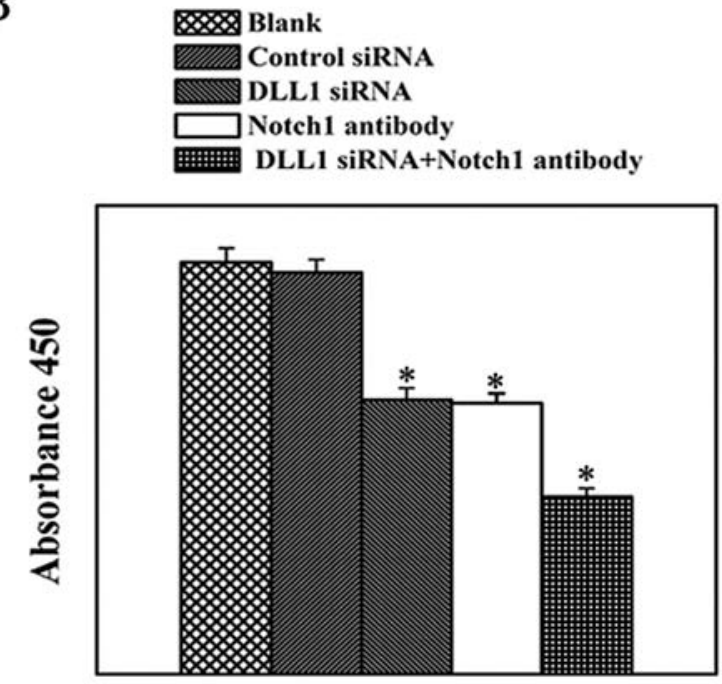

Figure 6. Knocking down of DLL1 and blocking of Notch1 receptors induced U251MG cell apoptosis and growth inhibition. U251MG cells cultured in 12and 96-well plates, were divided into five groups with three and six replicate wells respectively. Besides two groups as blank control and control siRNA groups, three groups were pretreated with DLL1 siRNA, Notch1 antibody and two joint measures respectively. (A) After $72 \mathrm{~h}$ flow Cytometry assay was performed with three replicate wells cells in 12-well plate using Annexin V-RPE and 7-AAD double staining. Annexin $\mathrm{V}^{-}$and 7-AAD-cells were used as controls. Annexin $\mathrm{V}^{+}$ and 7-AAD cells displayed apoptotic and Annexin $\mathrm{V}^{+}$and 7-AAD ${ }^{+}$cells were necrotic. (B) Columns indicate percents of apoptosis ratio improved by three kinds of treatment, which were worked out by minus blank or control siRNA groups. Compared with blank group, blocking Notch1 receptors improved apoptosis ratio $13.52 \pm 1.27 \%$, compared with control siRNA group, knocking down DLL1 and joint measure induced the proportion of apoptosis $15.15 \pm 1.38 \%$ and $41.32 \pm 1.97 \%$, respectively. Bars, \pm SE. Statistically significant differences from blank or control group were observed; "P $<0.05$. (C) WST-1 cell proliferation assay with cells in six replicate wells in a 96-well plate. Columns, mean of 450-nm absorbance; bars, \pm SE. Statistically significant differences from blank or negative control groups were observed; ${ }^{*} \mathrm{P}<0.05$.

glioma. We found there was no apoptosis or proliferation difference between down-regulation of DLL1 and blocking Notch1 receptors in U251MG cells. In the loop between Notch1 and DLL1, Notch1 and DLL1 interact with each other. Downregulation of DLL1 would affect the activation of Notch1 although Notch1 may be affected by other ligands (17). Adversely, down-regulation of Notch1 would induce less expression of DLL1. Both down-regulation of Notch1 and DLL1 would break the positive feedback loop. That may be the reason there was no difference between down-regulation of Notch1 and DLL1. It implies that DLL1 is an important target point for glioma treatment, not only because DLL1 was one of the ligands of Notch1, but also DLL1 was included in the juxtacrine loop. In addition, the joint knocking down of DLL1 and blocking Notch1 receptors induced much more apoptosis and growth inhibition than the sum of a single factor, which shows that the two measures cooperate with each other and have a synergistic role. So Notch1 and DLL1, as two ends of the feedback loop, could become a combined therapeutic target. 
Previous studies have found a juxtacrine loop between Notch receptor and $\Delta$ ligand in the formation of the nervous system in Drosophila and vertebrates, which worked as lateral inhibition (11). The lateral inhibition works as a negative feedback loop. Neural precursor cells express $\Delta$ strongly and the $\Delta$ ligand activates Notch in the neighbors, then the activation of Notch in these cells inhibits not only their differentiation, but also their expression of $\Delta(18)$. However in this study, what we found in glioma cells was opposite to the previous findings described above. U251MG expresses DLL1 which activates Notch1 in its neighbor, and then Notch1 induces more expression of DLL1 as a positive feedback. Despite the different phenomenon of DLL1-Notch1 feedback loop between tumor genesis and neural development, the difference of the mechanisms still needs further research.

In conclusion, Notch1 induced enhanced expression of DLL1 in U251MG glioma cells and there may be a positive feedback loop between Notch1 and DLL1. Inhibiting either member of the feedback loop induces U251MG cell apoptosis and inhibits proliferation. Down-regulating both members of the loop would produce of a stronger effect on apoptosis and proliferation which shows potential as a joint therapeutic target.

\section{Acknowledgements}

We are grateful to Xiasi Bio for the kind gift of Notch1 plasmid. This work is supported by the China Natural Science Foundation (Proj. No. 30672165 and 30772231 and 30872657) and Jiangsu Province's Medical Major Talent program (Proj.No. RC2007061).

\section{References}

1. Ma A, Boulton M, Zhao B, Connon C, Cai J and Albon J: A role for notch signaling in human corneal epithelial cell differentiation and proliferation. Invest Ophthalmol Vis Sci 48: 3576-3585, 2007.

2. Adam J, Myat A, Le Roux I, Eddison M, Henrique D, IshHorowicz D and Lewis J: Cell fate choices and the expression of Notch, Delta and Serrate homologues in the chick inner ear: parallels with Drosophila sense-organ development. Development 125: 4645-4654, 1998

3. Yu J, Poulton J, Huang YC and Deng WM: The hippo pathway promotes Notch signaling in regulation of cell differentiation, proliferation, and oocyte polarity. PLoS One 3: E1761, 2008

4. Djalilian AR, Namavari A, Ito A, Balali S, Afshar A, Lavker RM and Yue BY: Down-regulation of Notch signaling during corneal epithelial proliferation. Mol Vis 14: 1041-1049, 2008.
5. Kimura K, Satoh K, Kanno A, Hamada S, Hirota M, Endoh M, Masamune A and Shimosegawa T: Activation of Notch signaling in tumorigenesis of experimental pancreatic cancer induced by dimethylbenzanthracene in mice. Cancer Sci 98: $155-162,2007$.

6. Kanamori M, Kawaguchi T, Nigro JM, Feuerstein BG, Berger MS, Miele L and Pieper RO: Contribution of Notch signaling activation to human glioblastoma multiforme. J Neurosurg 106: 417-427, 2007

7. Milner LA and Bigas A: Notch as a mediator of cell fate determination in hematopoiesis: evidence and speculation. Blood 93: 2431-2448, 1999

8. Fox V, Gokhale PJ, Walsh JR, Matin M, Jones M and Andrews PW: Cell-cell signaling through NOTCH regulates human embryonic stem cell proliferation. Stem Cells 26: 715-723, 2008.

9. Katoh $\mathrm{M}$ and Katoh $\mathrm{M}$ : Integrative genomic analyses on HES/HEY family: Notch-independent HES1, HES3 transcription in undifferentiated ES cells, and Notch-dependent HES1, HES5, HEY1, HEY2, HEYL transcription in fetal tissues, adult tissues, or cancer. Int J Oncol 31: 461-466, 2007.

10. Purow BW, Haque RM, Noel MW, Su Q, Burdick MJ, Lee J, Sundaresan T, Pastorino S, Park JK, Mikolaenko I, Maric D, Eberhart CG and Fine HA: Expression of Notch-1 and its ligands, Delta-like-1 and Jagged-1, is critical for glioma cell survival and proliferation. Cancer Res 65: 2353-2363, 2005

11. Castro B, Barolo S, Bailey AM and Posakony JW: Lateral inhibition in proneural clusters: cis-regulatory logic and default repression by Suppressor of Hairless. Development 132: 3333$3344,2005$.

12. Zheng $X$ and Zhu X: Application of antibody blocking membrane receptor in NSC research. Heilongjiang Med Phar 25: 8-9, 2002

13. Livak KJ and Schmittgen TD: Analysis of relative gene expression data using real-time quantitative PCR and the 2 (Delta Delta C (T)) method. Methods 25: 402-408, 2001.

14. Zhang Y, Wang Z, Ahmed F, Banerjee S, Li Y and Sarkar FH: Down-regulation of Jagged-1 induces cell growth inhibition and S phase arrest in prostate cancer cells. Int J Cancer 119: 2071-2077, 2006.

15. Schutte B, Nuydens R, Geerts H and Ramaekers F: Annexin V binding assay as a tool to measure apoptosis in differentiated neuronal cells. J Neurosci Methods 86: 63-69, 1998.

16. Ito H, Aoki H, Kühnel F, Kondo Y, Kubicka S, Wirth T, Iwado E, Iwamaru A, Fujiwara K, Hess KR, Lang FF, Sawaya R and Kondo S: Autophagic cell death of malignant glioma cells induced by a conditionally replicating adenovirus. J Natl Cancer Inst 98: 625-636, 2006

17. Kiernan AE, Cordes R, Kopan R, Gossler A and Gridley T: The Notch ligands DLL1 and JAG2 act synergistically to regulate hair cell development in the mammalian inner ear. Development 132: 4353-4362, 2005

18. Cabrera CV: Lateral inhibition and cell fate during neurogenesis in Drosophila: the interactions between scute, Notch and Delta. Development 110: 733-742, 1990. 